\title{
Verschiedenes
}

\section{Suchaufruf: Tausendfüßler}

Bei der Auswertung der Fänge aus Bodenfallen oder Ausleseapparaturen fallen Tausendfüßler s. I. (Diplopoden, Chilopoden, Pauropoden und Symphylen) in oft nicht unbedeutenden Mengen an. Bevor dieses Tiermaterial venworfen wird oder in einer Schrankecke in Vergessenheit gerăt, würde ich mich freuen, es zu erhalten.

Grundsätzlich bin ich an Material aus aller Welt interessiert. Bezüglich Mitteleuropa interessieren mich besonders Fänge aus dem Alpenraum und den Mittelgebirgen.

Sollte der eine oder andere Kollege bei seinen Exkursionen Tausendfüßler für mich mitsammein, wäre ich dafür dankbar.

Jörg Spelda, Zoologisches Institut und Zoologisches Museum, Abteilung für Phylogenetische Systematik, Martin-Luther-King-Platz 3, D-W-2000 Hamburg 13

privat: Laienweg 33, D-W-7300 Esslingen, Tel. 0711/374029

\section{Personelles}

Dr. Luis Acosta, Catédra de Zoologial, Universidad Nacional de Cordoba, Argentinien, weilt ab 1. April 1991 im Rahmen eines Stipendiums des Deutschen Akademischen Austauschdienstes für ein Jahr am Insitut für Zoologie der Universität Mainz. Herr Acosta beschäftigt sich mit südamerikanischen Weberknechten, vor allem Gonyoleptidae.

Dr. Manfred Grasshoff C.I.D.A.-Korrespondent für Deutschland. Nach der Vereinigung der beiden deutschen Staaten besteht keine Notwendigkeit mehr, die Arachnologen der alten und der neuen Bundesländer durch je einen Korrespondenten beim C.I.D.A. in Paris zu vertreten. 
Dr. Manfred Grasshoff (Frankfurt/M.) und Dr. Peter Sacher (Wittenberg Lutherstadt), die über Jahre die Amtsgeschäfte für West- bzw. Ostdeutschland führten, einigten sich daher auf eine gemeinsame deutsche Interessenvertretung, die seit Jahresbeginn von Dr. Grasshoff wahrgenommen wird. Er ist unter der Anschrift

Dr. Manfred Grasshoff, Forschungsinstitut Senckenberg, Senckenberganlage 25, D-W-6000 Frankfurt/M. 1

bzw. telefonisch unter der Nummer 069-7542-267 zu erreichen. 